\title{
Murciélagos insectívoros aéreos en un paisaje ganadero del piedemonte llanero colombiano
}

\section{Aerial insectivorous bats in a cattle ranching landscape in the Colombian foothills}

\author{
Orlando Fabián Hernández Leal ${ }^{(i D)} \square$, Francisco Sánchez ${ }^{(D)} \square$, Diego J. Lizcano ${ }^{(D)} \square$
}

\section{Resumen}

La ganadería en el piedemonte llanero colombiano está ampliamente distribuida y se desconoce su impacto sobre los murciélagos insectívoros aéreos. Monitoreamos métodos acústicos pasivos a estos murciélagos en un sistema silvopastoril (SSP) plantado con Mimosa trianae, un sistema ganadero convencional dominado por pasto (SC) y un bosque (B) en el piedemonte llanero colombiano. La composición de especies del B se diferenció de las otras coberturas, mientras que no hubo diferencias entre SC y SSP. Hubo mayor riqueza en el SC que en las otras coberturas, mientras que no hubo diferencias entre el B y el SSP. Myotis nigricans, Peropteryx macrotis, Saccopteryx leptura, Molossus cf. rufus, Eptesicus I y Cormura brevirostris tuvieron la mayor actividad en el paisaje, y su uso del espacio dependió de la cobertura. La mayoría de las especies usaron el SC que las otras coberturas. En conclusión, la heterogeneidad espacial asociada al paisaje ganadero estudiado tiene efectos tanto en la estructura del ensamble como en las respuestas de las especies que lo conforman.

Palabras clave. Agroecosistemas. Bioacústica. Chiroptera. Orinoquia. Sistema silvopastoril.

\begin{abstract}
Cattle ranching is widely spread in the foothills of the Eastern Cordillera of Colombia, and its impact on aerial insectivorous bats is unknown. We used passive-acoustic monitoring of these bats in a silvopastoral system (SSP) planted with Mimosa trianae, a conventional livestock system dominated by pasture (SC) and a forest (B) in the Eastern Cordillera foothill of Colombia. Species composition of B differed from SC and SSP, whereas there were no differences between SC and SSP. Species richness was higher in the SC than in the other land covers, whereas there were no differences between B and SSP. Myotis nigricans, Peropteryx macrotis, Saccopteryx leptura, Molossus cf. rufus, Eptesicus I and Cormura brevirostris had the highest activity in the landscape, and their habitat use depended on land cover. Most bat species used SC more than the other land covers. We found that the spatial heterogeneity associated with the studied cattle ranching landscape affects both the structure of the bat ensemble and the responses of its species.
\end{abstract}

Key words. Agroecosystems, Bioacustics, Chiroptera, Habitat use, Orinoquia, Silvopastoral. 


\section{Introducción}

La ganadería es una de las principales actividades causantes de la transformación del paisaje en el planeta (Ramankutty et al., 2006), y se estima que aproximadamente un tercio de la superficie terrestre del planeta está destinada para la ganadería y la agricultura (Ellis et al., 2010). En Colombia la situación es similar, y se ha estimado que un $30 \%$ del territorio está dedicado a la ganadería, aproximadamente 34 millones de hectáreas, y más del 77 \% de esa área sufre algún grado de erosión (IDEAM \& UDCA, 2015). Así, la expansión ganadera genera una transformación de las coberturas naturales, incrementa la presión sobre los recursos naturales y tiene un efecto negativo sobre la biodiversidad (Alkemade et al., 2013; Dettenmaier et al., 2017). La ganadería está entre las primeras causas de pérdida de biodiversidad en Colombia, y en el piedemonte llanero amenaza los ecosistemas de bosque (Romero et al., 2008; Etter et al., 2017). Por todo lo anterior, se ha resaltado la necesidad de estrategias para conservar la alta diversidad del país, aun en esos ambientes altamente perturbados (Moreno et al., 2018).

Para contrarrestar los efectos negativos de la ganadería convencional, extensiva y basada principalmente en el uso de pastos exóticos, se ha sugerido el uso de los sistemas silvopastoriles, SSP (Calle et al., 2012), que integran el uso de árboles o arbustos con pastos para el ganado (Murgueitio et al., 2013). En comparación con los sistemas convencionales de ganadería, la cobertura heterogénea de los SSP puede facilitar la recuperación y mejoramiento de suelos, al mejorar el flujo de nutrientes y los ciclos locales del agua, (Alonso, 2011; Naranjo et al., 2012). En términos de los efectos sobre la biodiversidad, los arbustos y cercas vivas de los SSP pueden funcionar como corredores que favorecen la presencia de especies de murciélagos, aves, escarabajos, mariposas y fauna del subsuelo (Harvey et al., 2005; Mahecha, 2002).

En Colombia, los estudios sobre murciélagos en áreas ganaderas se han realizado en el Caribe, y se ha encontrado una mayor diversidad de Phyllostomidae en los SSP que en los sistemas convencionales (Ballesteros, 2015). También, una especie de murciélago frugívoro ha mostrado mejor condición corporal en los SSP que en sistemas convencionales, y algunas especies pueden acumular metales pesados producto de la adición de agroquímicos a los SSP (Chacón-Pacheco \& Ballesteros-Correa, 2019; Racero-Casarrubia et al., 2017). Así, es limitada la información sobre murciélagos insectívoros aéreos, i.e., que se alimentan de insectos en el aire (Wilson, 1979).
Algunos murciélagos insectívoros aéreos toleran ambientes altamente modificados y allí ayudan a conservar funciones ecosistémicas e incluso pueden prestar servicios ecosistémicos al controlar insectos que afectan negativamente los sistemas de producción (Boyles et al., 2011; Cleveland et al., 2006). Entre las herramientas para permitir que los murciélagos insectívoros persistan en ambientes rurales se encuentra el manejo de áreas arboladas (Guldin et al., 2007). Esto se debe a que las zonas arboladas naturales en paisajes altamente modificados por los humanos pueden afectar el uso del espacio de los murciélagos insectívoros aéreos, dado que pueden servir como refugio, fuentes de alimento o como corredores de tránsito (Barclay \& Kurta, 2007; Grindal \& Brigham, 1999). Además, en ambientes altamente intervenidos, los murciélagos hacen uso diferencial del espacio, según las coberturas presentes, ya que estas pueden afectar la disponibilidad de presas y las oportunidades de forrajeo (Avila-Flores \& Fenton, 2005; Jung \& Kalko, 2010). Así en diferentes localidades del neotrópico, se ha encontrado que cambios en el paisaje producto de la actividad agropecuaria pueden afectar el uso del espacio por los murciélagos insectívoros aéreos (Estrada et al., 2006; Williams-Guillén \& Perfecto, 2011).

Las comunidades de murciélagos en la base de los Andes orientales de Colombia se caracterizan por tener un rico ensamble de especies insectívoras aéreas (Ramírez-chaves et al., 2013; Sánchez, 2017); ensamble (Ramírez \& Gutiérrez-Fonseca, 2016) corresponde a un grupo filogenéticamente relacionado, i.e., Chiroptera, que explota un recurso similar, i. e., insectos aéreos. Entender cómo los ensambles de murciélagos insectívoros aéreos responden a las prácticas ganaderas puede servir para adoptar estrategias de manejo que reduzcan el impacto de esta actividad económica. Por ello, estudiamos un ensamble de murciélagos insectívoros aéreos en el piedemonte llanero colombiano, comparando un sistema ganadero convencional (SC), i. e., pastizal, un sistema silvopastoril (SSP) dominado por Mimosa trianae, y un remanente de bosque nativo. Nuestra primera hipótesis es que la riqueza y composición de especies de murciélagos insectívoros difieren entre las coberturas. Esto se debería a que el incremento en la densidad de obstáculos asociados a la cantidad de árboles presenta para los murciélagos problemas mecánicos por la necesidad de maniobrar, y problemas de percepción por la necesidad de detectar y evitar los obstáculos (Fenton, 1990). En consecuencia, la densidad de obstáculos en un ambiente se relaciona con la variación en la morfología de las alas y de las señales de ecolocalización usadas por murciélagos insectívoros, ocupando diferentes espacios 
(Aldridge \& Rautenbach, 1987; Thies et al., 1998). Nuestra segunda hipótesis es que los murciélagos insectívoros aéreos hacen un uso diferencial de las coberturas. Esto debido a las razones explicadas anteriormente, y porque en México se encontró una mayor actividad de murciélagos en fragmentos de bosques que en pastizales (Estrada et al., 2004), mientras que en Estados Unidos se halló una relación positiva entre la actividad y la distancia entre árboles (Gerth \& Chelsvig, 2008).

\section{Materiales y métodos}

Área de estudio. El piedemonte llanero colombiano es una subregión de la Orinoquia que se ubica en la base de la Cordillera Oriental de los Andes (Rangel-Ch. et al., 2011) y ha sido intensamente transformada por la actividad humana. Realizamos este estudio en la Finca La Rosania, ubicada en el municipio de San Luis de Cubarral, departamento del Meta ( $3^{\circ} 49^{\prime} 25.84^{\prime \prime} \mathrm{N} 73^{\circ}$ $50^{\prime} 2.87$ " W, 623 m s.n.m, Figura 1). El municipio tiene una precipitación anual de 2000-4000 mm y una temperatura anual de $20-27^{\circ} \mathrm{C}$ (Callejas Ávila, 2017; González Rey, 2017). A lo largo del año la precipitación presenta un régimen unimodal biestacional, con bajas precipitaciones entre diciembre y febrero, $\mathrm{y}$ altas precipitaciones entre marzo y noviembre (Datos IDEAM de 10 años, estación: 32060030).

La ganadería y la producción de leche son las principales actividades productivas de la finca. En el momento del estudio, los pastizales eran dominados por el género Brachiaria, cubrían aproximadamente 32.5 ha, y tenían árboles dispersos con alturas menores a $7 \mathrm{~m}$. El SSP estaba conformado por árboles de falso yopo Mimosa trianae, tenía un área de 1.23 ha, una edad de siembra de 2 a 3 años, y los árboles estaban sembrados cada 5 metros y tenían hasta $5 \mathrm{~m}$ de altura. Los pastos en el SSP eran del género Brachiaria, y allí se removían las ramas secas del suelo, para que no interfirieran el desplazamiento del ganado. El bosque de piedemonte de la finca tenía un área de 106.7 ha, con un estrato arbóreo de 12-25 m de altura, un estrato de arbolitos de 5-12 m de altura y un estrato arbustivo de 1.5-5 m de altura, según la descripción de Rangel \& Velásquez (1997).

Muestreo acústico. Realizamos en 2017 un muestreo estratificado, con nueve noches en febrero (baja precipitación), diez noches en junio, cuatro noches en octubre y una noche en noviembre (alta precipitación). En total, obtuvimos 24 noches efectivas de grabación (288 horas), i.e., 8 noches efectivas y 96 horas por cobertura. Usamos un detector ultrasónico SM4BAT FS con un micrófono externo SMM-U1 (Wildlife Acoustics ${ }^{\circledR}$ Maynard, MA, USA), El detector se instaló en seis sitios de grabación por cada cobertura. En cada punto de muestreo ubicamos el detector con el micrófono a $3 \mathrm{~m}$ sobre el nivel del suelo, en un ángulo de $45^{\circ}$ respecto a la superficie del suelo y apuntando hacia el interior de la cobertu$\mathrm{ra}$, desde las 18:00 $\mathrm{h}$ hasta las 06:00 h del día siguiente. Configuramos el detector para registrar frecuencias entre 16 y $120 \mathrm{kHz}$, con una tasa de muestreo de 256 $\mathrm{kHz}$, una ganancia de $12 \mathrm{~dB}$ y una longitud máxima de archivo de grabación de 15 segundos. El detector estuvo activo todo el tiempo de instalación, pero solo grabó cuando registró señales emitidas en el rango de frecuencias programado. Los archivos se guardaron en formato WAV sin compresión.

Dado que no existen librerías de referencia para la Orinoquia colombiana, realizamos identificaciones manuales siguiendo claves acústicas y literatura (Jung et al., 2014; Arias-Aguilar et al., 2018; López-Baucells et al., 2016; Zamora-Gutiérrez et al., 2016; Zurc et al., 2017) y comparando los siguientes parámetros: forma del pulso de ecolocalización, alternancia, frecuencia inicial, final, máxima, mínima, de máxima energía, amplitud, duración e intervalo entre pulsos. Analizamos las grabaciones en el programa BatSound-Sound Analysis V.4.2., y para las mediciones usamos el programa Raven Pro V.1.6 (The Cornell Lab of Ornithology). En ambos programas usamos una transformada rápida de Fourier ("fast Fourier transform") de 512 muestras con una ventana tipo Hamming. Dado que la configuración visual del programa afecta la percepción de los pulsos de ecolocalización, usamos los mismos valores en los parámetros para todas las grabaciones analizadas: 1500 ms por imagen, threshold $=10$, amplitud de contraste $=3$, en BatSound; ajuste de brillo = 63, ajuste de contraste $=67 \mathrm{y}$ overlap $=95 \%$ en Raven Pro. Los pulsos de ecolocalización no asignados a una especie se registraron como sonotipos, i. e., un grupo de sonidos con las mismas características y que asumimos correspondían a la misma especie (Bader et al., 2015). Los registros con baja intensidad o con pulsos muy tenues $(<45 \mathrm{~dB})$ se clasificaron como "no identificados".

Los murciélagos insectívoros aéreos emiten señales de ecolocalización que pueden ser agrupadas en tres fases: búsqueda, aproximación y terminal (Schnitzler \& Kalko, 2001). La emisión de fases de búsqueda representa el desplazamiento o la búsqueda de presas, las fases de aproximación representan la detección de los objetos o presas, y la fase terminal ("feeding buzz") 


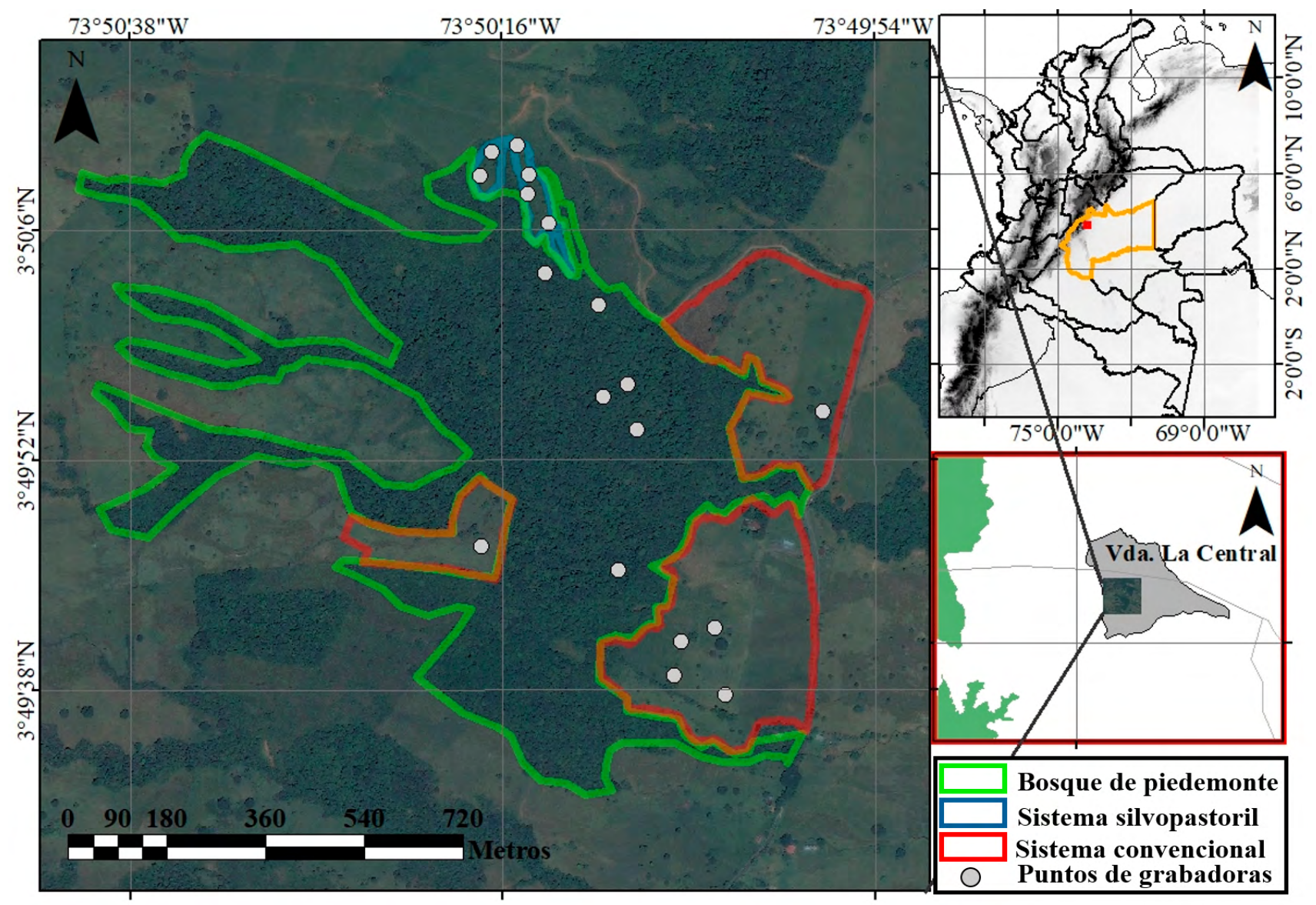

Figura 1. Ubicación del detector (puntos blancos) para el muestreo acústico de la actividad de murciélagos insectívoros aéreos en tres coberturas de un paisaje ganadero del municipio de Cubarral, departamento del Meta, Colombia.

Figure 1. Location of the detector (white dots) for the acoustic sampling of the activity of aerial insectivorous bats in three land covers of a livestock landscape in Cubarral, Meta, Colombia.

indica intentos de los murciélagos para capturar presas. Por ello, evaluamos el uso de las coberturas contando el número de fases de búsqueda y las fases terminales de cada especie por noche, como un indicador de la actividad del murciélago en cada cobertura (Sánchez, 2011).

Análisis estadísticos. Para soportar las identificaciones asignadas, realizamos análisis de función discriminante (DFA) (Parsons \& Jones, 2000) por cada familia, con base en diez grabaciones de cada identificación y medimos tres pulsos por secuencia, de los cuales se extrajeron los parámetros antes mencionados (Anexo 1). Medimos el total de archivos paras las identificaciones que no superaron las diez grabaciones y seleccionamos grabaciones de las diferentes coberturas. También medimos los pulsos bajos, medios y altos para las especies que presentan alternancia en los pulsos de ecolocalización. De acuerdo a lo sugerido por López-Baucells et al., (2016), agrupamos en Eptesicus I las posibles especies de este género.

Para determinar la variación de la composición de murciélagos entre las coberturas e identificar, las especies y los sonotipos causantes de la variación, usamos un análisis de similaridad ANOSIM, con 9999 permutaciones y un análisis SIMPER (Clarke, 1993). Estos análisis se realizaron en el programa Past V.3. También construimos curvas de acumulación de especies con la función "specaccum" del paquete "vegan", para evaluar posibles diferencias en la riqueza de especies entre coberturas, en el programa R versión 3.4 (Oksanen et al., 2019; R Core Team, 2019). 
Evaluamos el uso de coberturas de los murciélagos insectívoros con un modelo lineal generalizado (GLM) con una distribución de Poisson (Sheskin, 2004; Zar, 2010). Usamos esta opción, dada la naturaleza de conteo de los datos. En el modelo se incluyó el número de fases de búsqueda de todas especies registradas como variable dependiente, mientras que el tipo de cobertura y el mes entraron como variables independientes. También, realizamos el mismo GLM usando como variable dependiente el número de fases terminales de todas las especies registradas. Para detectar diferencias entre coberturas y meses, realizamos contrastes con un ajuste de Bonferroni (Sheskin, 2004). Debido a la cercanía entre los sitios de muestreo, aplicamos la prueba de autocorrelación espacial con el índice de Moran, usando una matriz de pesos con las distancias entre los cinco vecinos más cercanos. El análisis se realizó con la función "moran.test" del parquete "spdep" de R, usando los cinco vecinos más cercanos de cada punto de muestreo (Bivand \& Wong, 2013; R Core Team, 2019). Evaluamos, además, el uso del espacio para las especies con mayor número de fases de búsqueda y terminales, i.e., especies con más de 150 fases de búsqueda en todo el muestreo. Para ello, realizamos un GLM con una distribución de Poisson con la misma estructura del análisis general del uso de coberturas.

\section{Resultados}

Análisis del ensamblaje. En total analizamos 10845 archivos de audio, correspondientes a $28.8 \mathrm{~GB}$ de información, y con 6387 archivos con pulsos de ecolocalización de murciélagos. Encontramos 23 sonotipos representadas en cuatro familias, Además, siete identificaciones a nivel de sonotipo y algunos registros de baja intensidad, que no pudieron ser identificados (Anexo 2). Para la familia Molossidae, identificamos seis sonotipos, dos a nivel de familia, dos a nivel de género y dos a nivel de especie. Para Emballonuridae, identificamos un sonotipo a nivel de género y cinco a nivel de especie. Para la familia Vespertilionidae, identificamos seis sonotipos, dos a nivel de familia, dos a nivel de género y dos a nivel de especie. Para Thyropteridae, identificamos un sonotipo a nivel de género. En el SC, registramos 23 especies, de las cuales 14 fueron exclusivas de esa cobertura, mientras que en el B hubo 12 especies, seis exclusivas allí. Para el SSP registramos 15 especies, todas compartidas con el B o el SC. En consecuencia, la composición de murciélagos de acuerdo con el ANOSIM varió significativamente entre las coberturas $(R=0.234, p<0.01)$. La composición del $\mathrm{B}$ fue significativamente diferente a la de las otras dos coberturas (B vs. SC: $p<0.001$; B vs. SSP: $p=0.042)$, mientras que esto no ocurrió entre el SSP y el SC $(p=0.058)$. El SIMPER indicó que entre el B y el SC hubo una disimilitud del $74.9 \%$, entre el B y el SSP, del $72.7 \%$ y entre el SSP y el SC, del $52.7 \%$. Las especies que aportaron en mayor proporción a la disimilitud entre las coberturas fueron: Eptesicus I, Myotis nigricans, Molossus cf. rufus, Peropteryx macrotis, Cormura brevirostris, Saccopteryx leptura (Anexo 3). Por otra parte, la riqueza de especies fue mayor en el SC que en las otras coberturas, mientras que no hubo diferencias entre el B y el SSP (Figura 2).

Análisis general del uso de las coberturas. No hubo efecto de la cercanía de los sitios de grabación en la frecuencia de las fases de búsqueda y terminales (desviación estándar $[$ d.e $]=-0.253 ; p=0.600 ;$ d.e $=-0.338$; $p=0.368$, respectivamente). Por ello no se incluyó este factor en los análisis subsecuentes. La frecuencia de fases de búsqueda y terminales fue afectada por el tipo de cobertura $(g . l=2$; d.e $=999.683 ; p<0.001 ; g . l=2$; d.e $=$ 73.480; $p<0.001$, respectivamente) (Figura 3), siendo más usado el SC ( $\bar{x}=395.9$ fases de búsqueda; $\bar{x}=376$ fases terminales), seguido del SSP $(\bar{x}=232.7$ fases de búsqueda $\bar{x}=30.1$ fases terminales $)$ y el $B(\bar{x}=139.2$ fases búsqueda; $\bar{x}=15.7$ fases terminales). También, la frecuencia de las fases de búsqueda y terminales dependió de los meses $(g . l=3$; d.e $=275.196 ; p<0.001 ; g . l=3$; d.e $=231.158 ; p<0.001$, respectivamente) (Figura 3 ), siendo mayores los valores en junio ( $\bar{x}=294.7$ fases de búsqueda; $\bar{x}=52.2$ fases terminales), seguidos de octubre ( $\bar{x}=219.0$ fases de búsqueda; $\bar{x}=22.9$ fases terminales) y por último los de febrero $(\bar{x}=198.8$ fases búsqueda; $\overline{\mathrm{x}}=15.0$ fases terminales).

Uso de las coberturas por especie. Todas las especies examinadas, dos de la familia Vespertilionidae (Eptesicus I, M. nigricans), tres Emballonuridae ( $P$. macrotris, S. leptura y C. brevirostris), y un Molossidae (M. cf. rufus), presentaron diferencias significativas en el uso de las tres coberturas, en términos del número de fases de búsqueda y terminales (en todos los MLG's $p<0.01)$. En todos los casos fue mayor el uso del SC, seguido por el SSP y por el B (Figura 4), excepto para $S$. leptura. Para este embalonúrido, de acuerdo con las fases de búsqueda, el uso fue $\mathrm{SSP}>\mathrm{SC}=\mathrm{B}$, mientras que, según las fases terminales, fue $\mathrm{SSP}>\mathrm{SC}>\mathrm{B}$. Por otra parte, el mes de muestreo también tuvo un efecto significativo sobre el número de fases de búsqueda y fases terminales en las seis especies (en todos los MLG's $p<0.05$; Figura 4), con excepción del número de fases terminales de $M$. nigricans ( $g . l=3 ;$ d.e $=7.332 ; p>0.05)($ Anexo 4$)$. 


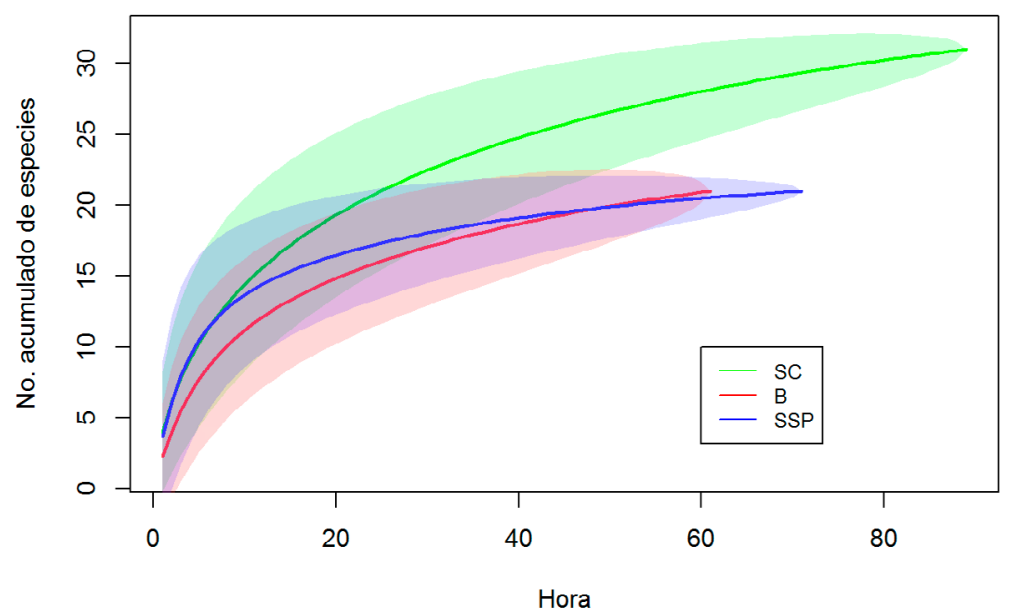

Figura 2. Curvas de acumulación de especies de murciélagos insectívoros aéreos de tres tipos de coberturas en un paisaje ganadero en Cubarral, Meta, Colombia. Las horas de grabación se usaron como unidades de muestreo. La línea continua representa la riqueza estimada para cada cobertura y el espacio sombreado de cada línea corresponde al intervalo de confianza del $95 \%$.

Figure 2. Species-accumulation curves of aerial insectivorous bats recorded in three types of land cover in a cattle ranching landscape in $\mathrm{Cu}$ barral, Meta, Colombia. Recording hours were used as sampling units. The solid line represents the estimated richness for each cover and the shaded area corresponds to the $95 \%$ confidence interval.

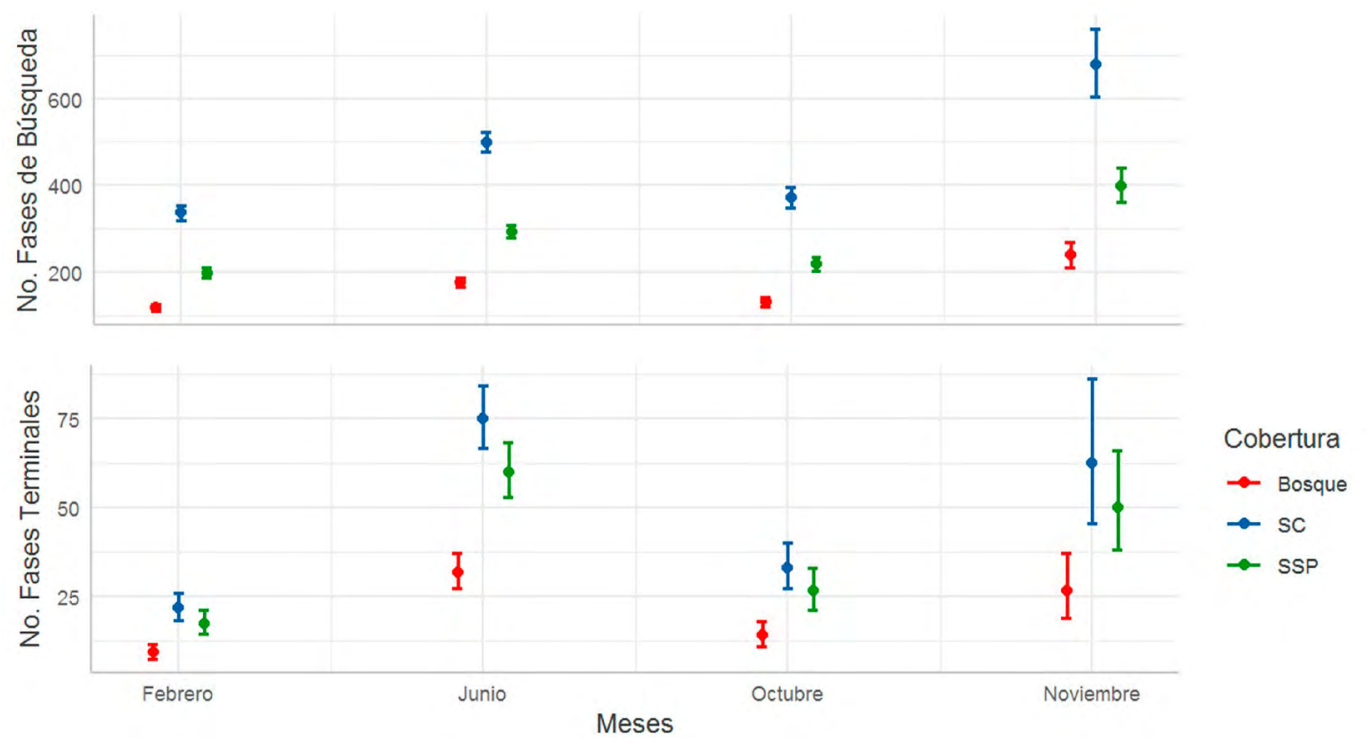

Figura 3. Uso de coberturas en los meses de muestreo por los murciélagos insectívoros aéreos en un paisaje ganadero en Cubarral, Meta, Colombia. El uso se midió en número de fases de búsqueda por noche (panel superior). Los círculos son promedios y los bigotes, una desviación estándar.

Figure 3. Use of land covers types by aerial insectivorous bats on the sampling months in a cattle ranching landscape in Cubarral, Meta, Colombia. Land cover use was measured as number of search phases per night (upper panel). Circles are averages and whiskers are one standard deviation. 

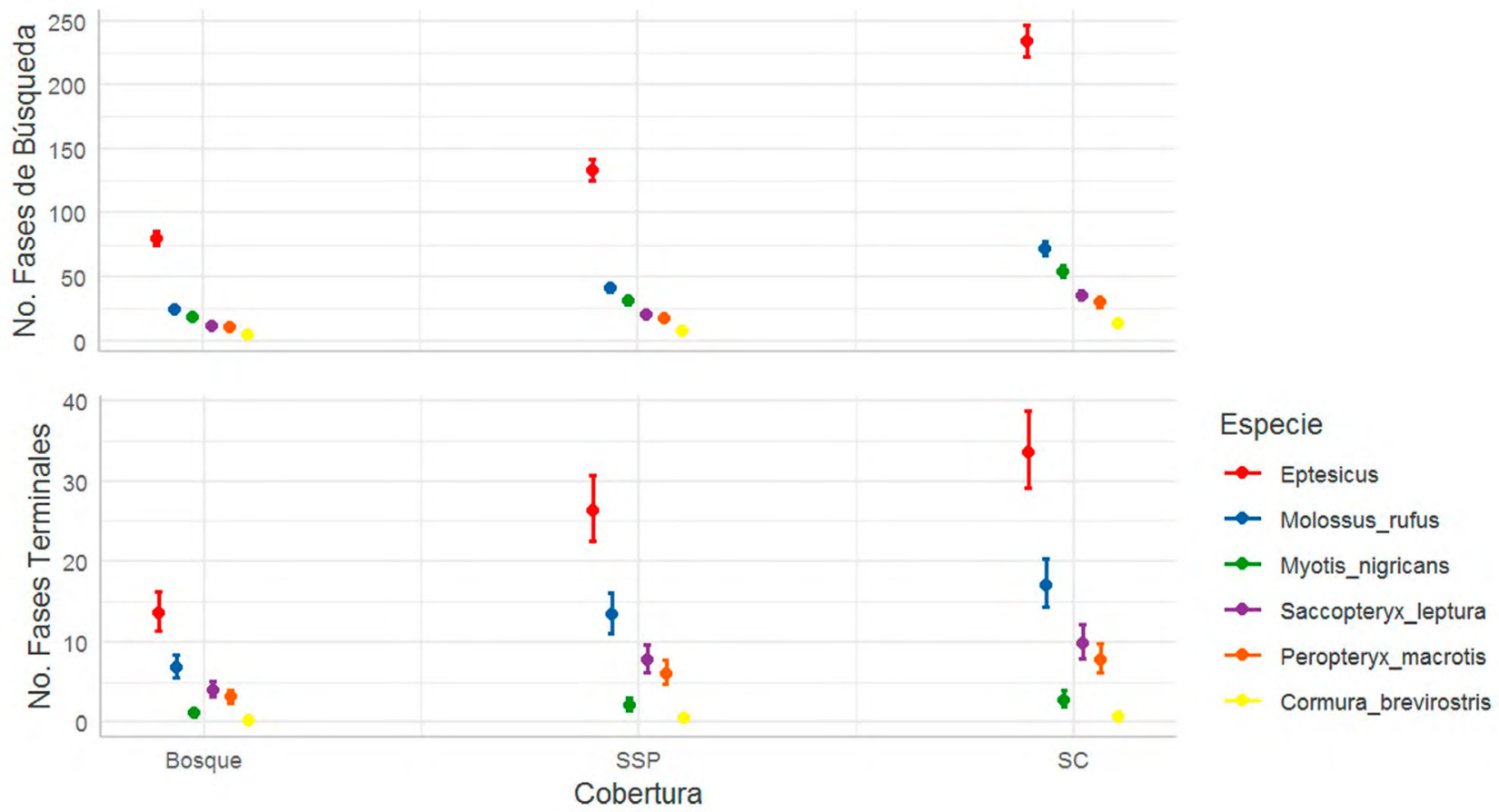

Figura 4. Uso de coberturas por seis especies de murciélagos insectívoros aéreos en un paisaje ganadero en Cubarral, Meta, Colombia. El uso se midió según el número de fases de búsqueda (panel superior), y terminales (panel inferior), por noche. Los círculos son promedios y los bigotes, una desviación estándar.

Figure 4. Use of land cover type by six aerial insectivorous bats species in a cattle ranching landscape. Use was measured in number of search phases (upper panel) and feeding buzzes (lower panel) per night. Circles are averages and whiskers are one standard deviation.

\section{Discusión}

Análisis del ensamble. De acuerdo a lo esperado, encontramos diferencias en la composición y riqueza de especies entre las coberturas del paisaje ganadero. La composición encontrada en el bosque, fue significativamente diferente a la encontrada en el SSP, pero no detectamos diferencias entre esta última cobertura y el sistema convencional. Así, en términos de composición el SSP es más parecido a un SC que al bosque. Nuestros resultados del SIMPER, que evalúan la disimilaridad teniendo en cuenta la representación de cada especie por cobertura (Mumby, 2001), también indican que las especies con mayor número de registros son las que aportan más a la diferenciación entre las coberturas. La mayoría de esas especies tienen marcadas diferencias en el uso de cada cobertura, lo que explica la diferenciación. Además, la riqueza en el SC, fue seguida por la del SSP y la del bosque. Así, los árboles espaciados y su bajo porte, $\sim 5 \mathrm{~m}$, en el SSP, permiten que especies de espacios abiertos usen esta cobertura. Por otra parte, la densidad de obstáculos y altura de los árboles en el bosque actúan como un filtro para algunas especies de murciélagos insectívoros aéreos, es decir que hay un efecto de la estructura de la vegetación sobre el ensamblaje de murciélagos insectívoros (Jung et al., 2012; Marques et al., 2016). En otras palabras, estos resultados concuerdan con el hecho que hay murciélagos insectívoros aéreos que prefieren el interior del bosque, y otros que prefieren los espacios abiertos (Jung et al., 2012; Schnitzler \& Kalko, 2001), pero a nivel de composición no hay una clara diferencia en la cobertura con una densidad de obstáculos intermedia, i. e., el SSP. A nivel de riqueza, el SSP no se diferenció del B, esta variable fue mayor en el SC lo que contrasta con la literatura que documenta la relación positiva entre la complejidad de la estructura vegetal y la riqueza de especies (August, 1983). Así, nuestros resultados indican que la disminución del área boscosa producto de la expansión de los SC en la zona de estudio ha tenido un efecto negativo sobre las especies propias del bosque. Inventarios de murciélagos del piedemonte de 
la Cordillera Oriental sugieren que estos ensambles son ricos en murciélagos insectívoros (Ramírez-Chaves et al., 2013; Sánchez, 2017), lo que es confirmado por nuestros resultados. Probablemente esta riqueza, que implica redundancias ecológicas (Walker, 1992), permite que haya especies que hoy, después de la intensa perturbación, puedan ocupar los ambientes abiertos creados por la ganadería.

Por otra parte, otro factor que puede influir en los resultados obtenidos está relacionado con el método de muestreo. La naturaleza abierta del SC permite que el micrófono capte señales sin interferencias a mayor distancia, debido a que hay una menor atenuación de los llamados en ambientes con ramas y vegetación (Kraker-Castañeda et al., 2013). Adicionalmente, la imposibilidad logística para ubicar el micrófono a más de tres metros del suelo dentro del sotobosque probablemente no permitió capturar las señales que emiten los murciélagos insectívoros que vuelan por encima del dosel. Sin embargo, en otras áreas del neotrópico, y usando métodos acústicos, se ha podido establecer que los murciélagos insectívoros usan en mayor proporción los fragmentos de bosque que las áreas abiertas, por lo que estas herramientas parecen ser adecuadas para evaluar efectos como los presentados aquí (Estrada et al., 2004; Kraker-Castañeda et al., 2013).

Por otra parte, el uso de detectores ultrasónicos ha permitido ampliar considerablemente los inventarios de murciélagos neotropicales (Azofeifa et al., 2019; Ochoa et al., 2000; Zamora-Gutierrez et al., 2016). El listado de murciélagos insectívoros aéreos presentado en este trabajo es el primero obtenido por métodos acústicos para la Orinoquia, y sugiere la presencia de dos nuevos registros para la región: C. greenhalli y P. nasutus (Ramírez-Chaves et al., 2016; Solari et al., 2013). Se requieren exploraciones adicionales, con obtención de llamadas de referencia, y capturas con redes de dosel para confirmar estos hallazgos.

Uso de las coberturas. Los murciélagos insectívoros aéreos hicieron un uso diferencial de las coberturas evaluadas. El SC fue la cobertura con mayor uso, seguida del SSP y finalmente el bosque. Estos resultados concuerdan con otros estudios en áreas altamente perturbadas, en las que se ha registrado que la actividad general es afectada por la composición del paisaje (Gerth \& Chelsvig, 2008). Sin embargo, nuestros resultados contrastan con un estudio en México en el que los murciélagos insectívoros usaron más los fragmentos de bosque que las áreas abiertas (Estrada et al., 2004). En otro ejemplo de México, los murciélagos insectívoros no tuvieron diferencias en el uso de bosques y pastizales (Kraker-Castañeda et al., 2013). Así, en el futuro sería recomendable comparar diferentes sitios con SC, SSP y bosques para confirmar si el efecto reconocido en este trabajo corresponde a un patrón, o es una excepción asociada a las particularidades del sitio de estudio.

Detectamos un efecto asociado a los meses de muestreo sobre el total de fases de búsqueda y terminales, pero no encontramos una conexión con la estacionalidad de la lluvia en la región, excepto que el mes con menor actividad fue el periodo seco. Otros estudios también reportan un efecto de la temporalidad en el uso de hábitat de los murciélagos insectívoros y se ha argumentado que el efecto es explicado por el régimen de lluvias y su relación con la disponibilidad de insectos (Barros et al., 2014; Williams-Guillén \& Perfecto, 2011). Por lo tanto, estudios de mayor duración con una mejor representación de cada fase del ciclo hidrológico, y con mediciones de la abundancia de insectos en la zona, pueden ayudar a explicar mejor la variación temporal que detectamos.

Uso de las coberturas por especie. Las seis especies analizadas usaron de manera diferencial las tres coberturas. A excepción de $S$. leptura que, en términos de fases terminales, prefirió usar el SSP sobre el SC y el bosque, el uso de las otras cinco especies fue SC > SSP > Bosque. El uso de hábitat en los murciélagos insectívoros se relaciona con características particulares en la movilidad y las características de los pulsos de ecolocalización (Bader et al., 2015; Schnitzler \& Kalko, 2001). Así, cada especie de murciélago tiene un hábitat en el cual se desempeña mejor (Kalko et al., 2008; Marinello \& Bernard, 2014), y se pueden reconocer murciélagos adaptados a bosques y otros murciélagos adaptados a espacios abiertos, y cada uno de ellos usa en mayor proporción las coberturas que más le conviene (Estrada-Villegas et al., 2010; Estrada et al., 2004; Jung \& Kalko, 2010.

Para la familia Vespertilionidae, las especies con mayor actividad en el paisaje ganadero fueron Eptesicus I y $M$. nigricans. A diferencia de las demás especies neotropicales del género Myotis, M. nigricans prefiere los ambientes abiertos (Fenton \& Bogdanowicz, 2002; Siemer et al., 2001), lo que concuerda con nuestros resultados. Por otra parte, se ha indicado que las especies del género Eptesicus tienden a tener una carga alar baja, una relación de aspecto media y modulaciones en las frecuencias emitidas, que les permite tener una alta maniobrabilidad, un vuelo suave y reconocer obstáculos de manera eficiente (Bader et al., 2015; Norberg \& Rayner, 1987). 
Estas características son apropiadas para espacios de borde o cerrados, lo que contrasta con lo encontrado en este estudio. Así, nuestros resultados probablemente son una evidencia de la versatilidad de los Eptesicus. La captura de especímenes de este género en el área de estudio debe permitir una descripción más detallada de sus características y así ayudar a resolver estas dudas.

En general, los Emballonuridae neotropicales son capaces de detectar presas en el borde y claros de los bosques (Jung et al., 2014; Schnitzler \& Kalko, 2001). Sin embargo, $P$. macrotis tiene una carga alar media y una relación de aspecto alta, que le permite volar rápido y aprovechar espacios abiertos (Estrada-Villegas et al., 2010; Marinello \& Bernard, 2014; Marques et al., 2016) lo que concuerda con nuestros resultados. Las especies $S$. leptura y $C$. brevirostris tienen una carga alar baja y una relación de aspecto alta, que les permite tener una alta maniobrabilidad a vuelos relativamente rápidos y aprovechar los bordes de los bosques y los claros (Bader et al., 2015; Kalko et al., 2008; Marques et al., 2016). A pesar de esto C. brevirostris usó con mayor frecuencia las zonas abiertas, mientras que $S$. leptura usó más el SSP. Esto podría relacionarse con el hecho de que C. brevirostris (machos $7.9 \mathrm{~g}$; hembras $10.15 \mathrm{~g}$ ) es más grande que $S$. leptura (machos $4.4 \mathrm{~g}$; hembras $5.7 \mathrm{~g}$ ) (Eisenberg, 1989). Adicionalmente, es interesante que en el ensamble la única que parece preferir el SSP sea $S$. leptura, y en el futuro debería evaluarse si esto facilita su coexistencia con las otras especies.

El único Molossidae analizado fue M. cf. rufus. Molossus rufus tiene una relación de aspecto y carga alar media, pulsos de ecolocalización largos y con baja frecuencia, y en algunos casos con alternancia, que les permite volar rápidamente y ser eficientes en capturar presas en zonas semi-abiertas (Bader et al., 2015; Marques et al., 2016). Esto concuerda con el bajo registro de actividad de este murciélago en el bosque.

De acuerdo a lo discutido, con base en la morfología alar y en las señales de ecolocalización, varias de las especies de murciélagos insectívoros registrados deberían ser capaces de explotar de manera eficiente el SSP, pero su actividad se concentra principalmente en el SC. En el momento, solo podemos especular, pero las zonas de SSP y bosque tienen no solo más obstáculos, sino tal vez más sitios de percha para depredadores como búhos y halcones que cazan al atardecer o en la noche. Así, estudios futuros deberían explorar en conjunto el uso del espacio por depredadores de murciélagos que podrían explicar el mayor uso de las áreas abiertas
En conclusión, la heterogeneidad espacial del paisaje ganadero estudiado afectó la estructura del ensamble de murciélagos insectívoros aéreos, así como el uso de hábitat de algunas especies. Nuestros resultados resaltan la utilidad de los métodos bioacústicos para reconocer los efectos de los cambios a nivel de paisaje tanto sobre el ensamble, como sobre las respuestas de las especies que lo conforman. En la medida en que la industria agropecuaria sea más productiva y sostenible, el uso de técnicas de monitoreo acústico estandarizado podrá ser usado en áreas más extensas para proveer indicadores del impacto y de la sostenibilidad de la industria agrícola (Doohan et al., 2019).

\section{Agradecimientos}

Agradecemos a Humberto Castro de La Rosania, por permitir el acceso a su finca y su apoyo durante el trabajo de campo. A Gabriela Moreno, por el apoyo en campo. A Jefferson Sánchez, Daniela Martínez, Angélica Yantén y Carmen Rocha, por la asesoría en las identificaciones acústicas y los comentarios de forma en el documento. Tres evaluadores anónimos hicieron importantes comentarios que ayudaron a mejorar sustancialmente la calidad y estructura del documento. Este estudio fue financiado por el proyecto Ganadería Colombiana Sostenible en alianza entre el Fondo para el Medio Ambiente Global (GEF), el gobierno del Reino Unido (UK), la Federación Colombiana de Ganaderos (FEDEGAN), The Nature Conservancy (TNC), la Fundación Centro para la Investigación en Sistemas Sostenibles de Producción Agropecuaria (CIPAV) y el Fondo para la Acción Ambiental (FA), bajo la supervisión del Banco Mundial.

\section{Referencias}

Aldridge, H. D. J. N. \& Rautenbach, I. L. (1987). Morphology, echolocation and resource partitioning in insectivorous bats. Journal of Animal Ecology, 56(3), 763-778.

https:/ / doi.org/10.1644/11-MAMM-A-331.1

Alkemade, R., Reid, R. S., van den Berg, M., de Leeuw, J. \& Jeuken, M. (2013). Assessing the impacts of livestock production on biodiversity in rangeland ecosystems. Proceedings of the National Academy of Sciences of the United States of America, 110(52), 2090020905.

https:/ / doi.org/10.1073/pnas.1011013108 
Alonso, J. (2011). Los sistemas silvopastoriles y su contribución al medio ambiente. Revista Cubana de Ciencia Agrícola, 45(2), 107-115.

Arias-Aguilar, A., Hintze, F., Aguiar, L. M. S., Rufray, V., Bernard, E. \& Pereira, M. J. R. (2018). Who's calling? Acoustic identification of Brazilian bats. Mammal Research 63(3), 231-253.

https:/ / doi.org/10.1007/s13364-018-0367-z

Armenteras, D., Rodríguez, N., Retana, J. \& Morales, M. (2011). Understanding deforestation in montane and lowland forests of the Colombian Andes. Regional Environmental Change, 11(3), 693-705.

https:/ / doi.org/10.1007/s10113-010-0200-y.

August, P. V. (1983). The role of habitat complexity and heterogeneity in structuring tropical mammal communities. Ecological Society of America, 64(6), 1495-1507.

Avila-Flores, R. \& Fenton, M. B. (2005). Use of spatial features by foraging insectivorous bats in a large urban landscape. Journal of Mammalogy, 86(6), 1193-1204. https:/ / doi.org/10.1644/04-MAMM-A-085R1.1

Azofeifa, Y., Estrada-Villegas, S., Mavárez, J. \& Nassar, J. M. (2019). Activity of aerial insectivorous bats in two rice fields in the northwestern Llanos of Venezuela. Acta Chiropterologica, 21(1), 149-163.

https:/ / doi.org/10.3161/15081109acc2019.21.1.012

Bader, E., Jung, K., Kalko, E. K. V., Page, R. A., Rodríguez, R., \& Sattler, T. (2015). Mobility explains the response of aerial insectivorous bats to anthropogenic habitat change in the Neotropics. Biological Conservation, 186, 97-106.

https:/ / doi.org/10.1016/j.biocon.2015.02.028

Ballesteros, J. (2015). Efecto del Manejo Silvopastoril y Convencional de Ganadería Extensiva Sobre el Ensamblaje de Murciélagos Asociados a Fragmentos de Bosque Seco Tropical en Córdoba, Colombia. (Trabajo de grado). Bogotá D.C.: Pontificia Universidad Javeriana, Facultad de Ciencias. 220 pp.

Barclay, R., \& Kurta, A. (2007). Ecology and behavior of bats roosting in tree cavities and under bark. En Lacki, E.M., Hayes, J. \& Kurta, A. (Eds.), Bats in forests: conservation and management. Pp. 17-59. Baltimore.: The Johns Hopkins University Press.

Barros, M. A. S., Pessoa, D. M. A. \& Rui, A. M. (2014). Habitat use and seasonal activity of insectivorous bats (Mammalia: Chiroptera) in the grasslands of southern Brazil. Zoologia, 31(2), 153-161.

https:/ / doi.org/10.1590/S1984-46702014000200006

Bivand, R. S. \& Wong, D. W. S. (2013). Comparing implementations of global and local indicators of spatial associationtors of spatial association. Test, 27(3), 716-748.

https:/ / doi.org/10.1007/s11749-018-0599-x
Boyles, J. G., Cryan, P. M., McCracken, G. F. \& Kunz, T. K. (2011). Economic importance of bats in agriculture.pdf. Science, 332(April), 41-42.

https:// doi.org/10.1126/science.1201366

Calle, Z., Murgueitio, E. \& Chará, J. (2012). Integrating forestry, sustainable cattle-ranching and landscape restoration. Unasylva 239, 63(1), 31-40.

Callejas Ávila, Y. A. (2017). Evaluación morfométrica del caño Quebradón como aporte al ordenamiento ambiental del municipio de Cubarral, Meta. (Trabajo de grado). Bogotá D.C.: Universidad Santo Tomás, Facultad de Ciencias y Tecnologías.

Cárdenas, G. (1999). Comparación de la composición y estructura de la avifauna en diferentes sistemas de producción. Trabajo presentado Seminario Internacional sobre sistemas agropecuarios sostenibles. Cali, Colombia.

Chacón-Pacheco, J. \& Ballesteros-Correa, J. (2019). Mejor condición corporal de Artibeus lituratus en sistemas silvopastoriles que en sistemas convencionales de ganadería en Córdoba , colombia. Oecologia Australis.23(3), 589-605.

https:/ / doi.org/10.4257/oeco.2019.2303.16

Clarke, K. R. (1993). Non-parametric multivariate analyses of changes in community structure. Australian Journal of Ecology, 18(1), 117-143.

https:/ / doi.org/10.1111/j.1442-9993.1993.tb00438.x

Cleveland, C. J., Betke, M., Federico, P., Frank, J. D., Hallam, T. G., Horn, J., López, J. D., McCracken, G. F., Medellín, R. A., Moreno-aldez, A., Sansone, C. G., Westbrook, J. K. \& Kunz, T. H. (2006). Economic value of the pest control service provided by Brazilian free-tailed bats in south-central Texas. Frontiers in Ecology and the Environment, 4(5), 238-243.

https:/ / doi.org/10.1890/1540-9295(2006)004[0238:EVOTPC]2.0.CO;2

Dettenmaier, S. J., Messmer, T. A., Hovick, T. J. \& Dahlgren, D. K. (2017). Effects of livestock grazing on rangeland biodiversity: A meta-analysis of grouse populations. Ecology and Evolution, 7(19), 7620-7627. https:/ / doi.org/10.1002/ ece3.3287

Dias, L. E., Franco, A. A., Campello, E., De Faria, S. M. \& Da Silva, E. M. (1995). Leguminosas forestales: aspectos relacionados con su nutrición y uso en la recuperación de suelos degradados. Bosque, 16(1), 121-127.

Doohan, B., Fuller, S., Parson, S. \& Peterson, E. (2019). The sound of management: Acoustic monitoring for agricultural industries. Ecological Indicators, 96, 739-746.

https:// doi.org/10.1016/j.ecolind.2018.09.029. 
Eisenberg, J. (1989). Mammals of the Neotropics: The northern Neotropics. Chicago.: The Chicago Press. 449 pp.

Ellis, E. C., Goldewijk, K. K., Siebert, S., Lightman, D. \& Ramankutty, N. (2010). Anthropogenic transformation of the biomes, 1700 to 2000. Global Ecology and Biogeography, 19(5), 589-606.

https:/ / doi.org/10.1111/j.1466-8238.2010.00540.x

Estrada-Villegas, S., Meyer, C. F. J. \& Kalko, E. K. V. (2010). Effects of tropical forest fragmentation on aerial insectivorous bats in a land-bridge island system. Biological Conservation, 143(3), 597-608.

https:/ / doi.org/10.1016/j.biocon.2009.11.009

Estrada, A., Jiménez, C., Rivera, A. \& Fuentes, E. (2004). General bat activity measured with an ultrasound detector in a fragmented tropical landscape in Los Tuxtlas, Mexico. Animal Biodiversity and Conservation, 27(2), 5-13.

Estrada, C. G., Damon, A., Hernández, C. S., Pinto, L. S. \& Núñez, G. I. (2006). Bat diversity in montane rainforest and shaded coffee under different management regimes in southeastern Chiapas, Mexico. Biological Conservation, 132(3), 351-361.

https:/ / doi.org/10.1016/j.biocon.2006.04.027

Etter, A., Andrade, A., Saavedra, K., Amaya, P., Arevalo, P. \& Cortés, J. (2017). Lista roja de ecosistemas de Colombia (Vers. 2.0). Bogotá D.C. 6 pp. Pontificia Universidad Javeriana.

Fao \& LEAD. (2006). Livestock's long shadow: environmental issues and options. En Steinfield, H., Gerber, P., Wassenaar, T., Castel, V., Rosales, M. \& de Hann, C. (Eds.), Food and Agriculture Organization of the United Nations. Roma: FAO.

https:/ / doi.org/10.1007/s10666-008-9149-3

Fenton, M. (1990). The foraging behaviour and ecology of animal-eating bats. Canadian Journal of Zoology, $68,411-422$.

Fenton, M. B. \& Bogdanowicz, W. (2002). Relationships between external morphology and foraging behaviour: Bats in the genus Myotis. Canadian Journal of Zoology, 80(6), 1004-1013.

https:/ / doi.org/10.1139/z02-083

Gerth, S. \& Chelsvig, J. (2008). Bat activity in an urban landscape: patterns at the landscape and microhabitat scale. En Marzluff, J., Shulenberger, E., Endlicher, W., Alberti, M., Bradley, G. \& Ryan C. (Eds.), Urban Ecology. Pp: 437-453. Springer Science+Business Media.

González Rey, I. C. (2017). Caracterización socioeconómica de la población del municipio de Cubarral. (Tesis de grado). Villavicencio:Universidad de los Llanos,
Facultad de Ciencias Económicas, Escuela de Economía y Finanzas. 84 pp.

Grindal, S. \& Brigham, R. (1999). Impacts of forest harvesting on habitat use by foraging insectivorous bats at different spatial scales. Ecoscience, 6(1), 25-34. https:/ / doi.org/10.1080/11956860.1999.11952206.

Guldin, J., Emmingham, W., Carter, S. \& Saugey, D. (2007). Silvicultural practices and management of habitat for bats. En M. Lacki, J. Hayes, \& A. Kurta (Eds.), Bats in forests: conservation and management. Pp. 177-205. Baltimore.: The Johns Hopkins University Press.

Harvey, C. A., Villanueva, C., Villacís, J., Chacón, M., Muñoz, D., López, M., Ibrahim, M., Gómez, R., Taylor, R., Martínez, J., Navas, A., Sáenz, J., Sánchez, D., Medina, A., Vilchez, S., Hernández, B., Pérez, A., Ruiz, F., López, F., ... Sinclair, F. L. (2005). Contribution of live fences to the ecological integrity of agricultural landscapes. Agriculture, Ecosystems and Environment, 111(1-4), 200-230.

https:/ / doi.org/10.1016/j.agee.2005.06.011

IDEAM, \& UDCA. (2015). Sintesis del estudio nacional de la degradación de suelos por erosión en Colombia ( $\mathrm{p}$. 62). IDEAM-MADS.

Jung, K., Kaiser, S., Böhm, S., Nieschulze, J. \& Kalko, E. K. V. (2012). Moving in three dimensions: effects of structural complexity on occurrence and activity of insectivorous bats in managed forest stands. Journal of Applied Ecology, 49(2), 523-531.

https:/ / doi.org/10.1111/j.1365-2664.2012.02116.x

Jung, K. \& Kalko, E. K. (2010). Where forest meets urbanization: foraging plasticity of aerial insectivorous bats in an anthropogenically altered environment. Journal of Mammalogy, 91, 144-153.

https:/ / doi.org/10.1644/08-mamm-a-313r.1

Jung, K., Kalko, E. K. V. \& Von Helversen, O. (2007). Echolocation calls in Central American emballonurid bats: Signal design and call frequency alternation. Journal of Zoology, 272(2), 125-137.

https://doi.org/10.1111/j.1469-7998.2006.00250.x

Jung, K., Molinari, J. \& Kalko, E. K. (2014). Driving factors for the evolution of species-specific echolocation call design in new world free-tailed bats (Molossidae). PLOS ONE, 9(1).

https:/ / doi.org/10.1371/journal.pone.0085279

Kalko, E. K. V, Estrada-Villegas, S., Schmidt, M., Wegman, M. \& Meyer, C. F. J. (2008). Flying high-assessing the use of the aerosphere by bats. Integrative and Comparative Biology, 48(1), 60-73.

https://academic.oup.com/icb/article/48/1/ $60 / 625591$ 
Kraker-Castañeda, C., Santos-Moreno, A. \& García-García, J. L. (2013). Riqueza de especies y actividad relativa de murciélagos insectívoros aéreos en una selva tropical y pastizales en Oaxaca, México. Mastozoología Neotropical/Journal of Neotropical Mammals, 20(2), 255-267.

López-Baucells, A., Rocha, R., Bobrowiec, P., Bernard, E., Palmeirim, J. \& Meyer, C. (2016). Field Guide to Amazonian Bats. Manaus: INPA. 174 pp.

Mahecha, L. (2002). El silvopastoreo: una alternativa de producción que disminuye el impacto ambiental de la ganadería bovina. Revista Colombiana de Ciencias Pecuarias, 15(2), 226-231.

Marinello, M. M. \& Bernard, E. (2014). Wing morphology of neotropical bats: A quantitative and qualitative analysis with implications for habitat use. Canadian Journal of Zoology, 92(2), 141-147.

https:/ / doi.org/10.1139/cjz-2013-0127

Marques, J. T., Ramos Pereira, M. J. \& Palmeirim, J. M. (2016). Patterns in the use of rainforest vertical space by Neotropical aerial insectivorous bats: all the action is up in the canopy. Ecography, 39(5), 476-486.

https:/ / doi.org/10.1111/ecog.01453

Moreno, L. ., Rueda, C. \& Andrade, G. (2018). Biodiversidad 2017. Estado y tendencias de la biodiversidad continental de Colombia. Bogotá D.C.: Instituto de Investigación de Recursos Biológicos Alexander von Humboldt. 84 pp.

Mumby, P. J. (2001). Beta and habitat diversity in marine systems: a new approach to measurement, scaling and interpretation. Oecologia, 128, 274-280.

https:/ / doi.org/10.1007/s004420100643

Murgueitio, E. R., Chará, J. D., Solarte, A. J., Uribe, F., Zapata, C. \& Rivera, J. E. (2013). Agroforestería pecuaria y sistemas silvopastoriles intensivos (SSPi) para la adaptación ganadera al cambio climático con sostenibilidad. Revista Colombiana de Ciencias Pecuarias, 26, 313-316.

Naranjo, J., Cuartas, C., Murgueitio, E., Chará, J. \& Barahona, R. (2012). Balance de gases de efecto invernadero en sistemas silvopastoriles intensivos con Leucaena leucocephala en Colombia. Livestock Research for Rural Development, 24(8), 12. http:/ / www.Irrd. org/lrrd24/8/\%0Anara24150.htm

Norberg, U. M. \& Rayner, J. M. V. (1987). Ecological morphology and flight in bats (Mammalia; Chiroptera): wing adaptations, flight performance, foraging strategy and echolocation. Philosophical Transactions of the Royal Society of London. B, Biological Sciences, 316(1179), 335-427.

https:/ / doi.org/10.1098/rstb.1987.0030
Ochoa G, J., O’Farrell, M. J. \& Miller, B. W. (2000). Contribution of acoustic methods to the study of insectivorous bat diversity in protected areas from northern Venezuela. Acta Chiropterologica 2(2), 171-183.

Oksanen, J., Blanchet, F. G., Friendly, M., Kindt, R., Legendre, P., McGlinn, D., Minchin, P. R., O'Hara, R. B., Simpson, G. L., Solymos, P., Stevens H., M. H., Szoecs, E. \& Wagner, H. (2019). Vegan: Community Ecology Package.

Parsons, S., \& Jones, G. (2000). Acoustic identification of twelve species of echolocating bat by discriminant function analysis and artificial neural networks. Journal experimental Biology, 203(17), 2641-2656.

Pech-Canche, J. M., MacSwiney G., C. \& Estrella, E. (2010). Importancia de los detectores ultrasónicos para mejorar los inventarios de murciélagos Neotropicales. Therya, 1(3), 221-228.

https:/ / doi.org/10.12933/therya-10-17

R Core Team. (2019). R: The R Project for Statistical Computing. Recuperado el 29 de mayo de 2020, de

https:/ / www.r-project.org/

Racero-Casarrubia, J., Pinedo-Hernández, J., Ballesteros-Correa, J. \& Marrugo-Negrete, J. (2017). Metales pesados en especies de murciélagos (Quiróptera) asociados a una finca bajo manejo silvopastoril en el departamento de Córdoba, Colombia. Acta Zoológica Mexicana, 33(1), 45-54.

Ramankutty, N., Graumlich, L., Achard, F., Alves, D., Chhabra, A., DeFries, R. S., Foley, J., Geist, H., Houghton, R. A., Klein, K., Lambin, É., Millington, A., Rasmussen, K., Reid, R. S., \& Turner II, B. L. (2006). Global land cover change: Recent progress, remaining challenges. En E. Lambin \& H. Geist (Eds.). Land-Use and Land-cover Change: Local Processes with Global Impacts (Pp. 9-39). Berlin: Springer.

Ramírez, A., Gutiérrez-Fonseca, P. E. Sobre ensambles y ensamblajes ecológicos-respuesta a Monge Nájera. Revista de Biología Tropical, 64(2), 817-819.

Ramírez-Chaves, H. E., Noguera-Urbano, E. \& Rodríguez-Posada, M. E. (2013). Mamíferos (Mammalia) del departamento de Putumayo, Colombia. Revista de la Academia Colombiana de Ciencias Exactas, Físicas y Naturales, 37(143), 263-286.

Ramírez-Chaves, H., Suárez-Castro, A. \& González-Maya, J. F. (2016). Cambios recientes a la lista de los mamíferos de Colombia. Notas Mastozoológicas, 3(1), 120.

https:/ / doi.org/2382-3704

Rangel-Ch., J. O., Lowy-C., P. D., Aguilar-P., M., \& Garzón-C., A. (2011). Tipos de vegetación en Colombia: Una aproximación al conocimiento de la termino- 
logia fitosociológica, fitoecológica y de uso común. En Rangel-Ch., J. O., Lowy-C., P. D. \& M. Aguilar-P. (Eds.), Diversidad Biótica II (Pp. 89-207). Bogotá D.C.: Instituto de Ciencias Naturales, Universidad Nacional de Colombia.

Rangel-Ch., O. \& Velásquez, A. (1997). Métodos de estudio de la vegetación. En Rangel-Ch., O., Lowey, P. \& Aguilar, M. (Eds.), Diversidad Biótica II. 59-87 pp. Bogotá D.C.: Universidad Nacional de Colombia-Instituto de Ciencias Naturales, Instituto de hidrología, Meteorología y estudios Ambientales (IDEAM)-Ministerio del Medio Ambiente, Comité de Investigaciones y Desarrollo Científico-CINDEC.U.N, Academia Colombiana de Ciencias Exactas, Físicas y Naturales.

Romero, M., Cabrera, E. \& Ortiz, N. (2008). Informe sobre el estado de la biodiversidad en Colombia 2006-2007. Colombia: Instituto de Investigación de Recursos Biológicos Alexander von Humboldt.

Sánchez, F. (2011). La heterogeneidad del paisaje del borde norte de Bogotá (Colombia) afecta la actividad de los murciélagos insectívoros. Revista U.D.C.A. Actualidad y Divulgación Científica, 14(1), 71-80.

Sánchez, F. (2017). Bats of Villavicencio (Meta, Colombia): Preliminary assessment of their trophic diversity and ecosystem services. Boletin Cientifico del Centro de Museos, 21(1), 96-111.

https:/ / doi.org/10.17151/bccm.2017.21.1.8

Schnitzler, H.-U. \& Kalko, E. K. V. (2001). Echolocation by Insect-Eating Bats. BioScience, 51(7), 557-569.

https:/ / doi.org/0006-3568(2001)051[0557:EBIEB]2.0. $\mathrm{CO} ; 2$

Sheskin, D. (2004). Handbook of Parametric and Nonparametric Statistical Procedures (3rd ed). New York.: Chapman \& Hall/CRC. 1193 pp.

Siemers, B., Kalko, E. \& Schnitzler, H.-U. (2001). Echolocation behavior and signal plasticity in the Neo- tropical bat Myotis nigricans (Schinz, 1821) (Vespertilionidae): a convergent case with European species of Pipistrellus? Behavioral Ecology and Sociobiology, 50, 317-328.

Solari, S., Muñoz-Saba, Y., Rodríguez-Mahecha, J. V, Defler, T. R., Ramírez-Chaves, H. E. \& Trujillo, F. (2013). Riqueza, endemismo y conservación de los mamíferos de Colombia. Mastozoología Neotropical/ Journal of Neotropical Mammals, 20(2), 301-365.

Thies, W., Kalko, E. K. V. \& Schnitzler, H.-U. (1998). The roles of echolocation and olfaction in two Neotropical fruit-eating bats, Carollia perspicillata and C. castanea, feeding on Piper. Behavioral Ecology and Sociobiology, 42, 397-409.

https:/ / doi.org/10.1007/s002650050454

Walker, B.H (1992). Biodiversity and ecological redundancy. Conservation Biology, 6(1),18-23.

Williams-Guillén, K. \& Perfecto, I. (2011). Ensemble composition and activity levels of insectivorous bats in response to management intensification in coffee agroforestry systems. PLoS ONE, 6(1).

https:/ / doi.org/10.1371/journal.pone.0016502

Wilson, D. (1979). Bat faunas: a trophic comparison. Systematic Zoology, 22(1), 14-29.

Zamora-Gutiérrez, V., López-González, C., MacSwiney González, M. C., Fenton, B., Jones, G., Kalko, E. K. V., Puechmaille, S. J., Stathopoulos, V. \& Jones, K. E. (2016). Acoustic identification of Mexican bats based on taxonomic and ecological constraints on call design. Methods in Ecology and Evolution, 7(9), 1082-1091.

https:/ / doi.org/10.1111/2041-210X.12556

Zar, J. (2010). Biostatistical Analysis (D. Lynch (ed.); Fifth edit. Pearson education, Inc.

Zurc, D., Guillén-Servent, A. \& Solari, S. (2017). Chillidos de ecolocación de murciélagos Emballonuridae en una sabana xerófila semiseca del Caribe colombiano. Mastozoología Neotropical, 24(1), 201-218. 


\section{Anexo}

Anexo 1a: El $76.6 \%$ de las identificaciones realizadas para la familia Molossidae fueron asignadas correctamente de acuerdo a los análisis discriminantes. Dos funciones discriminantes explicaron el $97.5 \%$ de la varianza de los datos (Anexo 1b). Los parámetros acústicos con mayor peso en las dos funciones discriminantes fueron frecuencia final (Lambda de wilks $=0.006 ; p<0.001$ ), frecuencia de mayor energía (Lambda de wilks $=0.003 ; p<0.001$ ), ancho de banda (Lambda de wilks $=0.003 ; p<0.001$ ), intervalo de pulso (Lambda de wilks $=0.003 ; p<0.001) \mathrm{y}$ frecuencia máxima (Lambda de wilks $=0.002 ; p<0.001$ ) (Anexo 1c). Para la familia Emballonuridae, las funciones discriminantes asignaron correctamente el $93.2 \%$ de las identificaciones realizadas. Dos funciones discriminantes explicaron el 99. 2\% de la varianza de los datos (Anexo 1b). Los parámetros acústicos con mayor peso en las dos funciones discriminantes fueron: frecuencia de mayor energía (Lambda de wilks $=0.002 ; p=0.000$ ), frecuencia inicial (Lambda de wilks $=0.005 ; p<0.001$ ), Duración (Lambda de wilks $=0.003 ; p<0.001$ ), frecuencia máxima (Lambda de wilks $=0.003 ; p<0.001)$, frecuencia mínima (Lambda de wilks $=0.003 ; p<0.001$ ) e intervalo del pulso (Lambda de wilks $=0.002 ; p<0.001)($ Anexo $1 \mathrm{c})$. Para la familia Vespertilionidae y Thyropteridae, las funciones discriminantes asignaron correctamente el $82.3 \%$ de las identificaciones realizadas. Dos funciones discriminantes explicaron el $98.1 \%$ de la varianza de los datos (Anexo 1b). Los parámetros acústicos con mayor peso en las dos funciones discriminantes fueron: Frecuencia inicial (Lambda de wilks $=0.006 ; p<0.001$ ), frecuencia final (Lambda de wilks $=0.005 ; p<0.001$ ), frecuencia máxima (Lambda de wilks $=0.005 ; p<0.001$ ), frecuencia mínima (Lambda de wilks $=0.006 ; p<0.001$ ), Duración (Lambda de wilks $=0.007 ; p<0.001)$ e intervalo de pulso (Lambda de wilks $=0.005 ; p<0.001)$ (Anexo 1c). Para los sonotipos, las funciones discriminantes asignaron correctamente el $93.3 \%$ de las identificaciones realizadas. Dos funciones discriminantes explicaron el $95.4 \%$ de la varianza de los datos (Anexo 1b). Los parámetros acústicos con mayor peso en las dos funciones discriminantes fueron: frecuencia máxima (Lambda de wilks $=0.002 ; p<0.001$ ), ancho de banda (Lambda de wilks $=0.004 ; p<0.001)$, frecuencia inicial (Lambda de wilks $=0.003 ; p<0.001$ ), frecuencia final (Lambda de wilks $=0.002 ; p<0.001)$ y duración (Lambda de wilks $=0.002 ; p<0.001)($ Anexo 1c).

Anexo 1b. Parámetros generales de las funciones discrimantes por cada familia de murciélagos registrados por métodos bioacústicos en un paisaje ganadera del piedemonte llanero colombiano. En todos los análisis discrimantes se explicó más del 90 $\%$ de la variación. Var $(\%)$ = Porcentaje de variación explicada por función discriminante. Acum $(\%)$ = Porcentaje acumulado de las funciones discriminantes.

Annex 1b. General parameters of the discriminating functions for each family of bats recorded using bioacoustic methods in a cattle ranching landscape in the Andean foothills of Colombia. In all discriminating analyzes, more than $90 \%$ of the variation was explained. Var $(\%)=$ Percentage of variation explained by discriminant function. Accum $(\%)=$ Accumulated percentage of discriminant functions

\begin{tabular}{ccccc}
\hline \multicolumn{5}{c}{ Molossidae } \\
\hline f.d & Autovalor & Var (\%) & Acum. (\%) & Correlación canónica \\
\hline 1 & 54.8 & 93.7 & 93.7 & 0.991 \\
\hline 2 & 2.2 & 3.8 & 97.5 & 0.831 \\
\hline \multicolumn{7}{c}{ Emballonuridae } \\
\hline 2 & 104.4 & 98.1 & 98.1 & 0.995 \\
\hline & 1.2 & 1.1 & 99.2 & 0.735 \\
\hline 1 & 50.7 & Vespertilionidae & 0.990 \\
\hline 2 & 1.1 & 96.0 & 96.0 & 0.726 \\
\hline 1 & 2.1 & 98.1 & 0.987 \\
\hline 2 & 37.6 & Sonotipos & 0.930 \\
\hline
\end{tabular}


Anexo 1c. Parámetro acústicos mejor explicados en las funciones discriminates para cada familia. f.d = función discriminante.

Annex 1c. Best explained acoustic parameters in the discriminant functions for each family. f.d = discriminant function.

\begin{tabular}{lcc}
\hline \multicolumn{3}{c}{ Molossidae } \\
\hline Variable & f.d 1 & f.d 2 \\
\hline Frecuencia final & 0.960 & 0.121 \\
\hline Frecuencia Mayor energía & 0.807 & -0.391 \\
\hline Frecuencia máxima & 0,646 & -0.340 \\
\hline Ancho de banda & 0.117 & -0.297 \\
\hline Intervalo de pulso & -0.155 & 0.490 \\
\hline \multicolumn{1}{c}{ Emballonuridae } \\
\hline Frecuencia Mayor energía & 0.886 & \\
\hline Frecuencia final & 0.877 & -0.464 \\
\hline Frecuencia máxima & 0.834 & 0.248 \\
\hline Duración & -0.062 & 0.111 \\
\hline Frecuencia mínima & 0.597 & 0.289 \\
\hline Intervalo de pulso & -0.095 & 0.120 \\
\hline & & \\
\hline Frecuencia inicial & 0.903 & -0.206 \\
\hline Frecuencia mínima & 0.819 & -0.086 \\
\hline Frecuencia máxima & 0.531 & -0.135 \\
\hline Intervalo de pulso & -0.174 & 0.574 \\
\hline Duración & -0.250 & 0.580 \\
\hline Frecuencia final & 0.577 & 0.129 \\
\hline & 0.729 & -0.080 \\
\hline Frecuencia máxima & 0.656 & -0.109 \\
\hline Frecuencia final & & -0.512 \\
\hline Frecuencia inicial & & -0.214 \\
\hline Ancho de banda & & \\
\hline Duración & & \\
\hline & & \\
\hline
\end{tabular}


Anexo 1c. Agrupación de las especies de acuerdo a las funciones discriminantes generadas por familia. Juntamos las familias Vespertilionidae y Thyropteridae por la similitud en los parámetros acústicos.

Annex 1c. Species grouping based on the discriminant functions generated for each family. We pooled the data from the families Vespertilionidae and Thyropteridae due to the similarity in their acoustic parameters.
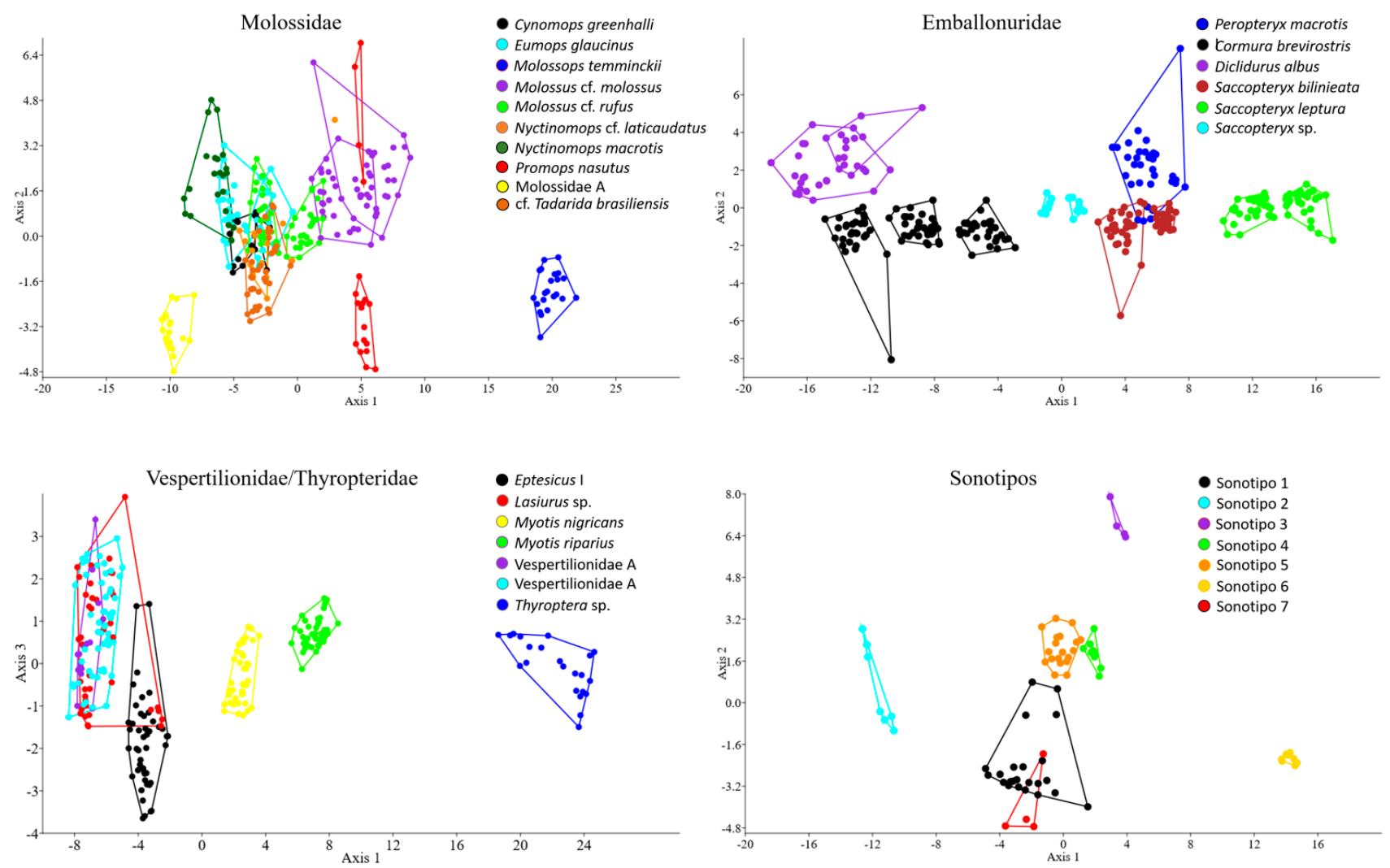
Anexo 2. Número de fases de búsqueda y de fases terminales de murciélagos insectívoros aéreos registradas en tres tipos de coberturas en un paisaje ganadero en el Meta, Colombia. En negrilla las especies elegidas para analizar el uso del espacio entre las coberturas evaluadas. Bosque de piedemonte $=\mathrm{B}$, sistema silvopastoril $=$ SSP y sistema convencional $=\mathrm{SC}$.

Annex 2. Number of search phases and terminal phases of aerial insectivorous bats recorded in three types of covers in a livestock landscape in Meta, Colombia. In bold the species chosen to analyze the use of the space between the evaluated covers. Piedmont forest = B, silvopastoral system $=$ SSP and conventional system $=$ SC.

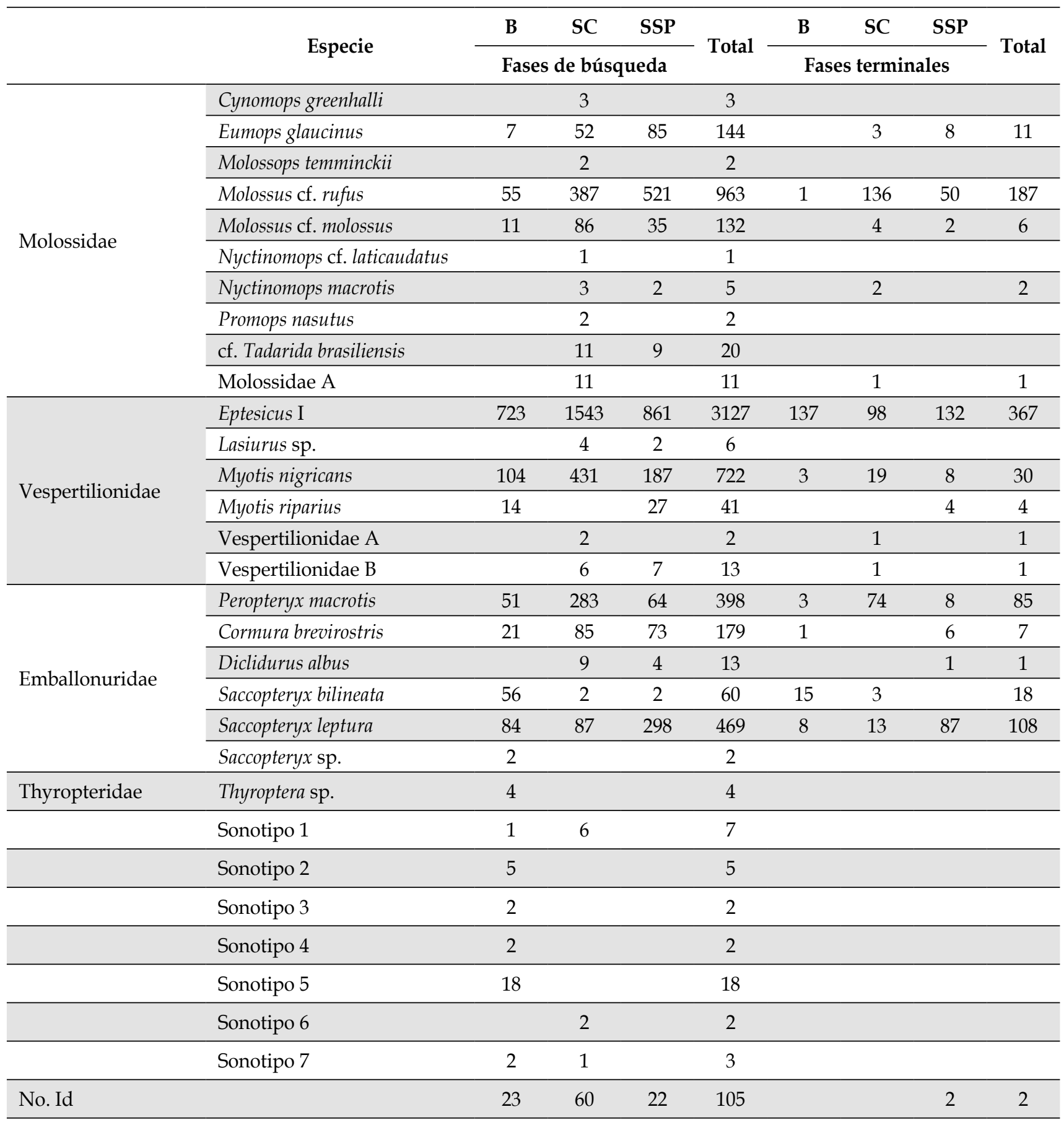


Anexo 3. Porcentaje de la contribución de cada especie de murciélago insectívoro aéreo a la medida de disimilaridad de Bray-Curtis entre tres tipos de coberturas en un paisaje ganadero en el Meta, Colombia. Bosque de piedemonte = B, sistema silvopastoril $=$ SSP y sistema convencional $=$ SC. Cont. Corresponde al porcentaje de disimilitud contribuido por cada especie. Acu. corresponde al porcentaje de disimilitud acumulado.

Annex 3. Percentage of the contribution of each species of aerial insectivorous bat to the Bray-Curtis dissimilarity measure between three types of cover in a livestock landscape in Meta, Colombia. Piedmont forest = B, silvopastoral system = SSP and conventional system =SC. Cont. Corresponds to the percentage of dissimilarity contributed by each species. Acu. corresponds to the percentage of dissimilarity accumulated.

\begin{tabular}{|c|c|c|c|c|c|c|c|c|}
\hline \multicolumn{3}{|l|}{ B vs SC } & \multicolumn{3}{|c|}{ B vs SSP } & \multicolumn{3}{|c|}{ SSP vs SP } \\
\hline \multicolumn{3}{|c|}{ Disimilitud $=74.9 \%$} & \multicolumn{3}{|c|}{ Disimilitud $=72.7 \%$} & \multicolumn{3}{|c|}{ Disimilitud $=52.7 \%$} \\
\hline Especie & $\begin{array}{c}\text { Cont. } \\
(\%)\end{array}$ & $\begin{array}{l}\text { Acu. } \\
(\%)\end{array}$ & Especie & $\begin{array}{c}\text { Cont. } \\
(\%)\end{array}$ & $\begin{array}{c}\text { Acu. } \\
(\%)\end{array}$ & Especie & $\begin{array}{c}\text { Cont. } \\
(\%)\end{array}$ & $\begin{array}{c}\text { Acu. } \\
(\%)\end{array}$ \\
\hline Eptesicus I & 44.6 & 44.6 & Eptesicus I & 40.4 & 40.4 & Eptesicus I & 38.7 & 38.7 \\
\hline Molossus cf. rufus & 16.0 & 60.6 & Molossus cf. rufus & 18.6 & 59.1 & Myotis nigricans & 14.7 & 53.4 \\
\hline Myotis nigricans & 12.5 & 73.1 & Saccopteryx leptura & 11.4 & 70.5 & Molossus cf. rufus & 13.8 & 67.2 \\
\hline Peropteryx macrotis & 9.6 & 82.7 & Myotis nigricans & 8.6 & 79.1 & Peropteryx macrotis & 9.6 & 76.8 \\
\hline Cormura brevirostris & 4.3 & 87.1 & Eumops glaucinus & 5.2 & 84.2 & Saccopteryx leptura & 8.8 & 85.6 \\
\hline Molossus cf. molossus & 2.7 & 89.8 & Peropteryx macrotis & 4.0 & 88.3 & Cormura brevirostris & 5.2 & 90.8 \\
\hline Saccopteryx leptura & 2.6 & 92.3 & Saccopteryx bilineata & 3.0 & 91.3 & Eumops glaucinus & 3.1 & 93.8 \\
\hline Eumops glaucinus & 2.2 & 94.6 & Cormura brevirostris & 2.6 & 93.8 & Molossus cf. molossus & 2.5 & 96.3 \\
\hline Saccopteryx bilineata & 2.1 & 96.7 & Molossus cf. molossus & 1.7 & 95.5 & Myotis riparius & 1.0 & 97.3 \\
\hline Sonotipo 5 & 0.6 & 97.3 & Myotis riparius & 1.4 & 96.9 & cf. Tadarida brasiliensis & 0.6 & 97.8 \\
\hline Myotis riparius & 0.6 & 97.9 & Sonotipo 5 & 1.0 & 97.9 & Vespertilionidae B & 0.4 & 98.3 \\
\hline cf. Tadarida brasiliensis & 0.2 & 98.1 & Vespertilionidae B & 0.5 & 98.3 & Diclidurus albus & 0.4 & 98.6 \\
\hline Molossidae A & 0.2 & 98.4 & cf. Tadarida brasiliensis & 0.4 & 98.8 & Molossidae A & 0.3 & 98.9 \\
\hline Diclidurus albus & 0.2 & 98.6 & Diclidurus albus & 0.3 & 99.1 & Lasiurus sp. & 0.2 & 99.1 \\
\hline Vespertilionidae B & 0.2 & 98.8 & Thyroptera sp. & 0.2 & 99.3 & Sonotipo 1 & 0.2 & 99.3 \\
\hline Sonotipo 1 & 0.2 & 98.9 & Saccopteryx sp. & 0.2 & 99.5 & Nyctinomops macrotis & 0.2 & 99.4 \\
\hline Thyroptera sp. & 0.1 & 99.1 & Sonotipo 2 & 0.2 & 99.6 & Saccopteryx bilineata & 0.2 & 99.6 \\
\hline Sonotipo 2 & 0.1 & 99.2 & Sonotipo 7 & 0.1 & 99.7 & Vespertilionidae A & 0.1 & 99.7 \\
\hline Lasiurus sp. & 0.1 & 99.3 & Lasiurus sp. & 0.1 & 99.8 & Sonotipo 6 & 0.1 & 99.7 \\
\hline Sonotipo 7 & 0.1 & 99.4 & Nyctinomops macrotis & 0.1 & 99.9 & Promops nasutus & 0.1 & 99.8 \\
\hline Saccopteryx sp. & 0.1 & 99.5 & Sonotipo 4 & 0.1 & 99.9 & Sonotipo 7 & 0.1 & 99.9 \\
\hline Nyctinomops macrotis & 0.1 & 99.6 & Sonotipo 3 & 0.1 & 100.0 & Cynomops greenhalli & 0.1 & 99.9 \\
\hline Vespertilionidae A & 0.1 & 99.7 & Sonotipo 1 & 0.0 & 100.0 & Molossops temminckii & 0.1 & 100.0 \\
\hline Sonotipo 6 & 0.1 & 99.7 & Vespertilionidae A & 0.0 & 100.0 & $\begin{array}{l}\text { Nyctinomops cf. } \\
\text { laticaudatus }\end{array}$ & 0.0 & 100.0 \\
\hline Promops nasutus & 0.1 & 99.8 & $\begin{array}{l}\text { Nyctinomops cf. } \\
\text { laticaudatus }\end{array}$ & 0.0 & 100.0 & Thyroptera sp. & 0.0 & 100.0 \\
\hline Cynomops greenhalli & 0.0 & 99.8 & Sonotipo 6 & 0.0 & 100.0 & Sonotipo 5 & 0.0 & 100.0 \\
\hline Molossops temminckii & 0.0 & 99.9 & Cynomops greenhalli & 0.0 & 100.0 & Sonotipo 4 & 0.0 & 100.0 \\
\hline Sonotipo 4 & 0.0 & 99.9 & Molossops temminckii & 0.0 & 100.0 & Sonotipo 3 & 0.0 & 100.0 \\
\hline Sonotipo 3 & 0.0 & 100.0 & Promops nasutus & 0.0 & 100.0 & Sonotipo 2 & 0.0 & 100.0 \\
\hline $\begin{array}{l}\text { Nyctinomops cf. } \\
\text { laticaudatus }\end{array}$ & 0.0 & 100.0 & Molossidae A & 0.0 & 100.0 & Saccopteryx sp. & 0.0 & 100.0 \\
\hline
\end{tabular}


Anexo 4. Efecto de las coberturas y los meses de muestreo en el uso de hábitat de seis especies de murciélagos insectívoros. Todas las especies hicieron un uso diferencial de las coberturas. El factor mes se agregó para controlar el efecto. $g . l=$ grados de libertad. d.e = desviación estándar.

Annex 4. Effect of the covers and the sampling months on the habitat use of six species of insectivorous bats. All species made differential use of the covers. The month factor was added to control the effect. g.l = degrees of freedom. d.e = standard deviation.

\begin{tabular}{|c|c|c|c|c|c|}
\hline Especie & Fase & Factores & g.1 & d.e & p \\
\hline \multirow{4}{*}{ Eptesicus I } & \multirow{2}{*}{ F. búsqueda } & Cobertura & 3 & 24602.040 & 0.000 \\
\hline & & Meses & 3 & 58.680 & 0.000 \\
\hline & \multirow{2}{*}{ F. terminal } & Cobertura & 3 & 1323.478 & 0.000 \\
\hline & & Meses & 3 & 131.697 & 0.000 \\
\hline \multirow{4}{*}{ M. nigricans } & \multirow{2}{*}{ F. búsqueda } & Cobertura & 3 & 3752.740 & 0.000 \\
\hline & & Meses & 3 & 268.940 & 0.000 \\
\hline & \multirow{2}{*}{ F. terminal } & Cobertura & 3 & 14.985 & 0.001 \\
\hline & & Meses & 3 & 7.333 & 0.063 \\
\hline \multirow{4}{*}{ P. macrotis } & \multirow{2}{*}{ F. búsqueda } & Cobertura & 3 & 1725.471 & 0.000 \\
\hline & & Meses & 3 & 69.496 & 0.000 \\
\hline & \multirow{2}{*}{ F. terminal } & Cobertura & 3 & 201.359 & 0.000 \\
\hline & & Meses & 3 & 35.010 & 0.000 \\
\hline \multirow{4}{*}{ S. leptura } & \multirow{2}{*}{ F. búsqueda } & Cobertura & 3 & 2076.397 & 0.000 \\
\hline & & Meses & 3 & 174.241 & 0.000 \\
\hline & \multirow{2}{*}{ F. terminal } & Cobertura & 3 & 259.868 & 0.000 \\
\hline & & Meses & 3 & 103.150 & 0.000 \\
\hline \multirow{4}{*}{ C. brevirostris } & \multirow{2}{*}{ F. búsqueda } & Cobertura & 3 & 455.088 & 0.000 \\
\hline & & Meses & 3 & 95.351 & 0.000 \\
\hline & \multirow{2}{*}{ F. terminal } & Cobertura & 3 & 26.389 & 0.000 \\
\hline & & Meses & 3 & 13.156 & 0.000 \\
\hline \multirow{4}{*}{ M. cf. rufus } & \multirow{2}{*}{ F. búsqueda } & Cobertura & 3 & 5688.115 & 0.000 \\
\hline & & Meses & 3 & 144.481 & 0.000 \\
\hline & \multirow{2}{*}{ F. terminal } & Cobertura & 3 & 623.733 & 0.000 \\
\hline & & Meses & 3 & 68.833 & 0.000 \\
\hline
\end{tabular}


Orlando Fabián Hernández Leal

Universidad de los Llanos

Villavicencio, Colombia

faleal1995@gmail.com

https:/ / orcid.org/0000-0003-1545-0349

\section{Francisco Sánchez}

Universidad de los Llanos

Villavicencio, Colombia

fsanchezbarrera@unillanos.edu.co

https:/ / orcid.org/0000-0001-8826-5692

\section{Diego Lizcano}

The Nature Conservancy

Bogotá, Colombia

diego.lizcano@tnc.org

https:/ / orcid.org/0000-0002-9648-0576

Murciélagos insectívoros aéreos en un paisaje ganadero del piedemonte llanero colombiano

Citación del artículo: Hernández-Leal, O.F., Sánchez, F. \& Lizcano, D. (2021). Murciélagos insectívoros aéreos en un paisaje ganadero del piedemonte llanero colombiano. Biota Colombiana, 22(1), 164-183.

https:/ / doi.org/10.21068/c2021.v22n01a11

Recibido: 31 de mayo 2020

Aceptado: 17 de noviembre 2020 